\title{
LA CARACTERIZACIÓN LEXICOGRÁFICA DE LA COMPLEMENTACIÓN DEL ADJETIVO INGLÉS Y SU TRATAMIENTO EN LOS DICCIONARIOS MONOLINGÜES DE APRENDIZAJE
}

\author{
Manuel Ramos Cortés \\ I.E.S. Joaquín Turina, Sevilla \\ RESUMEN \\ Una de las peculiaridades sintácticas del adjetivo, la selección de complementos propios de forma análoga al \\ verbo, aunque menos frecuente y de modo más limitado que éste, genera la búsqueda del desarrollo de una \\ tipología completiva para esta categoría que pueda, a su vez, generar aplicaciones lexicográficas adecuadas. \\ Los diccionarios monolingües de aprendizaje del inglés, con un ganado prestigio en la configuración de \\ modelos de complementación verbal y que ya intentaron tímidamente reflejar los comportamientos \\ completivos del adjetivo, son reclamados como marco idóneo para reflejar en su aparato de información \\ gramatical la complementación adjetiva en términos de igualdad con el tratamiento otorgado a la esfera \\ verbal. \\ Palabras clave: Gramática inglesa, sintaxis del adjetivo, lexicografía, uso del diccionario, lingüística aplicada \\ ABSTRACT \\ Adjective complementation and its lexicographic possibilities is one of the issues currently taken into \\ consideration by many scholars. It has given rise to an important line of investigation seeking for an \\ appropriate completive typology. English monolingual learning dictionaries, which originally included a \\ complete code system to reflect in their entries relevant patterns of verbal syntactical behaviour, are claimed \\ to be one of the most suitable frames to account for adjective complementation in similar terms as those \\ created for verbs. There is no reason why the rigour observed when dealing with verb-patterns cannot be \\ applied in an attempt to build up a system of information to users as far as adjectival complementation is \\ concerned. \\ Keywords: English grammar, Adjective syntax, lexicography, dictionary use, applied, linguistics.
}

\section{INTRODUCCIÓN.}

Una de las tendencias hacia las que con mayor frecuencia se orientan los estudios lingüísticos en los últimos tiempos es la búsqueda de aplicaciones lexicográficas idóneas para los fenómenos sintácticos objeto de investigación. Efectivamente, lexicografía y gramática se dan la mano a la hora de tratar de configurar ese terreno que reconocen como propio, cual es la caracterización sintáctico-semántica de las piezas léxicas. A pesar de que la tradición lexicográfica no ha estado exenta de controversia en torno a la conveniencia de considerar informaciones de índole gramatical en los diccionarios, lo cierto es que 
actualmente nadie duda de los beneficios que para los usuarios aporta la inclusión de la misma en estas obras. Destaca, sobre todo, uno: proporcionar la máxima información descriptiva y de uso de una lengua a los aprendices no nativos de ésta. Es ése, precisamente, el cometido de un tipo de diccionario lingüístico, los monolingües de aprendizaje (en adelante, DMA), cuyas especiales características lo configuran como un género propio y que en el caso de la lengua inglesa (los denominados diccionarios "learner's") han tenido un amplio desarrollo desde la primera mitad del pasado siglo.

Desde sus orígenes y a lo largo de su evolución ha destacado en ellos el enorme rigor seguido a la hora de reflejar de la forma más cómoda para el usuario los valores completivos de los verbos, pero, lamentablemente, como deseamos demostrar, en mucha menor medida, los de otras clases de palabra. Se aleja del propósito de nuestro trabajo entrar a describir la meticulosidad con la que los sucesivos editores han elaborado estas obras'; en cambio, sí queremos destacar la falta de ese mismo esmero por reflejar la sintaxis del adjetivo, toda vez que ésta demuestra poseer suficientes elementos de subcategorización susceptibles de ser catalogados en igualdad de condiciones que el verbo.

Pretendemos, pues, abordar en el presente trabajo una revisión de la complementación del adjetivo inglés y de cómo su caracterización completiva se ha dirigido mediante diversos criterios taxonómicos hacia el encuentro de utilidades lexicográficas efectivas. En este sentido, queremos analizar el tratamiento que los DMA clásicos dieron a este tema y como cobra actualidad la necesidad de retomar este género como terreno abonado para, de forma similar a como se trata la complementación verbal, reflejar también la tipología completiva de los predicados no verbales, en especial (aunque no sólo), del adjetivo.

Comenzaremos por resumir las teorías conducentes a caracterizar al adjetivo como predicación capaz de seleccionar sus propios complementos y dejar así fuera de duda su similitud con la categoría verbal. Analizaremos a continuación tres acercamientos significativos a una tipología completiva del adjetivo y su orientación hacia un lexicón sintáctico del mismo. Ello nos llevará a defender la validez de los DMA como marco idóneo para reflejar esa tipología en la catalogación léxica de forma análoga a como lo hacen con el verbo, para lo que

\footnotetext{
${ }^{1}$ Recomendamos el estudio de la evolución de este género llevada a cabo por Cowie (1999); asimismo, un interesante estudio del tratamiento de los modelos oracionales verbales en los DMA lo encontramos en Rizo Rodríguez (1992) y Dodd (1994).
} 
nos adentraremos a analizar el tratamiento que del adjetivo se hace en estas obras. Mantendremos que es posible formular modelos de conducta sintáctica del adjetivo similar a los planteados en el ámbito verbal, por lo que nos permitiremos sugerir algunos códigos que, junto a los ya utilizados en los diccionarios, puedan dar cuenta de los modelos sintagmáticos de aquella categoría. Hacerlo así enriquecería notablemente el contenido de los diccionarios lingüísticos para aprendices extranjeros, a la vez que desarrollaría un marco idóneo de aplicación lexicográfica en el ámbito de la investigación sintáctica.

\section{LA COMPLEMENTACIÓN DEL ADJETIVO}

\subsection{El adjetivo como predicación}

Tradicionalmente, sólo al verbo se le concedía la capacidad de seleccionar complementos; el adjetivo y el nombre, dentro de sus sintagmas, sólo tenían posibilidad de ser modificados (o, en el caso de este último, determinado). Existía cierta reticencia a aplicar criterios propios del dominio verbal fuera de ese contexto; téngase en cuenta que en su primera gramática Quirk et al. (1972) se referían a los dependientes del adjetivo y del nombre como su pre- o postmodification; es en Quirk y Greenbaum (1973) y, posteriormente, en Leech y Svartvik (1975) donde se emplea el término "complements of the adjective", aunque es en su obra monumental, Quirk et al. (1985), donde el grupo de gramáticos desarrollan la idea más pormenorizadamente. Actualmente, por influencia de la gramática generativa y de dependencias, en la mayoría de los estudios lingüísticos que tienen que ver con estas categorías ese enfoque está plenamente aceptado y sólo existen discrepancias en torno a los términos que emplear y la naturaleza de esas dependencias. Incluso, algunos autores ( $c f r$., Escandell Vidal, 1995 y Fernández Leborans, 2003) sólo se refieren a una única categoría de modificadores y, dentro de ellos, distinguen un tipo especial, seleccionado por el núcleo, que encuadran dentro de la denominación de complemento. Está, por tanto, fuera de duda la naturaleza predicativa de los sintagmas adjetivos, planteándose la controversia sólo en torno a los criterios para distinguir la naturaleza de sus dependientes. Tomaremos a continuación algunos de los argumentos que apoyan la capacidad completiva de esta categoría a partir de las ideas distintivas de la complementación verbal y veremos cómo se adapta a ellas el adjetivo.

La evidencia de complementación comúnmente aceptada para el verbo, la obligatoriedad y, alternativamente, la latencia, vuelven a servirnos en el sintagma adjetivo para poner de manifiesto la naturaleza completiva de algunos de los elementos que lo forman. Lo vemos, por ejemplo, en la construcción $\mathrm{He}$ is fond 
of him, frente a 'He is fond, en un claro caso de obligatoriedad 'fuerte' en el sentido descrito por Matthews (1981). Los adjetivos que necesitan completar su referencia semántica lo hacen seleccionando unos dependientes específicos vinculados al tipo de adjetivo que acompañan y excluyendo otros. Como en el caso de los verbos, puede decirse que los complementos forman parte de su valencia. Tal es el caso en la oración: She's very able to do the job, frente a *She's very able in/about/etc./doing the job, o 'She's very able to her friend. Es decir, el adjetivo able expresa de modo selectivo su propio régimen, aceptando unos elementos completivos y rechazando otros.

Señalemos, por otra parte, dos características de la complementación: por un lado, el sintagma adjetivo genera construcciones claramente atributivas (endocéntricas en la terminología bloomfieldiana), She's very keen/keen to do it, y exocéntricas, She's fond of him/"She's fond; por otro, finalmente, su naturaleza predecible en caso de elisión lo distingue de los modificadores ( $c f r$., We are ready to go / We are ready).

Las capacidades completivas de los adjetivos ofrecen una importante y útil posibilidad de clasificación sintáctica y lexicográfica más allá de criterios puramente nocionales, basados en la modificación del referente o la referencia, y del que tiene que ver con su posición y orden. Como señala Comesaña Rincón (2000: 175), "adjectival complementation is less numerous and complex than verbal complementation (...). And it is possible that, for this very reason, rigorous analysis of adjectival complementation is not to be found in lexicographical productions". Así, encontramos que para algunos estudios lexicográficos del adjetivo sólo cuentan su posición y sus cualidades semánticas (cfr., Krishnamurthy, 1987: 67). No obstante, el hecho de que los verbos superen en número y frecuencia de aparición a los adjetivos no puede convertirse en argumento para negarles su capacidad completiva; hay que tener en cuenta que, si bien es cierto que muchos adjetivos se bastan a sí mismos, ocurre igual con los llamados verbos intransitivos. Añádase a esto que los adjetivos sólo pueden llevar complementación en su posición predicativa (The violin is difficult to play), los adjetivos atributivos rechazan esa posibilidad ("A keen on chess child), salvo, como señala Comesaña Rincón (2000:161 nota), casos como easy y hard, "which do admit attributive position even displaying their respective complementation: That is an easy to study lesson. It is a hard to find parking spot". Los adjetivos en posición atributiva optan por desplazar la complementación (The violin is a difficult instrument to play), o posponer el sintagma verbal al nombre (The violin is an instrument (rather) difficult to play) (cfr., Quirk et al., 1985: 1220). 
Esa tendencia de las formas adjetivas complementadas a asumir posiciones distribucionales paralelas a las de los verbos, afianza la consideración de esta categoría como elemento predicativo. En línea con esa idea, Huddleston (1984: 183) aporta el siguiente argumento:

From a semantic point of view, fond is similar to love [in i: Ed loves Kim; ii: Ed is fond of Kim.] -they express the two-place semantic predicates "fond" and "love", which represent similar relations. From a syntactic point of view, however, they are very different, for fond is an adjective while love is a verb (...). Because fond is an adjective it cannot carry a tense inflection (...). In (ii) the tense inflection is carried by the verb be, and it is plausible to suggest that the only role of be here is precisely to carry the tense inflection -that it does not itself express a semantic predicate.

Como vemos, no es fácil ignorar las peculiaridades sintácticas de naturaleza completiva que rodean al adjetivo. Veamos, también, la reflexión que Quirk et al., (1985: 1221) aportan sobre el paralelismo con la esfera verbal:

There is a considerable parallel (...) between patterns associated with adjectives and those associated with monotransitive verbs. The chief difference is that adjectives cannot be followed by a noun phrase object. (...) We could describe adjectives [such as] averse to, conscious of, etc. as 'prepositional adjectives' comparable with prepositional verbs. We could moreover identify 'phrasal adjectives' (...) and 'phrasal-prepositional adjectives'.

Todos estos argumentos refuerzan la idea de un comportamiento predicativo del sintagma adjetival con un núcleo adjetivo capaz de regir dependencias de modo análogo al dominio verbal. El análisis de sus potencialidades completivas es relevante para la descripción sintáctica y su catalogación lexicográfica. Con ese objetivo, cobra importancia la búsqueda de una tipología completiva de la categoría adjetiva que permita su catalogación en un 'lexicon' y/o en los diccionarios convencionales.

Es esa aplicación lexicográfica la que nos interesa y, por su relevancia en este sentido, destacamos tres modelos de sistematización. Por un lado, Quirk et al. (1985) establecen un extenso catálogo de complementos y estructuras sintagmáticas que acompañan al adjetivo, que puede muy bien tomarse como referencia base en este tema. En segundo lugar, nos encontramos con la propuesta de tipología completiva transitiva del adjetivo, denominada así por su autor, expuesta por Comesaña Rincón (1998). Por último, tenemos el análisis valencial con finalidad lexicográfica que encontramos en Herbst (1984). Son, en suma, tres ejemplos significativos de la línea metodológica que, hoy por hoy, se 
refleja en los diccionarios o intentan recoger los trabajos de repertorios léxicosintácticos del adjetivo.

\subsection{El modelo de Quirk y su grupo}

En Quirk et al. (1985:1220 y sig.) se presenta un modelo de complementación del adjetivo articulado en torno a diecisiete estructuras básicas (el número es mayor si se tienen en cuenta algunos otros subgrupos especificados en la serie). De forma esquemática, podemos resumirlos en el siguiente cuadro (mantenemos la seriación y referencias originales):

[E1] Adjective complementation by a prepositional phrase.

He was worried about her reaction.

She was arware of her difficulties.

[E2] Adjective complementation by a that-clause.

[E2a] Adjectives with experiencer as subject.

We were confident that Karen was still alive.

Are you willing that he should be permitted to resign?

[E2b] Adjectives with anticipatory it as subject.

It is true that she never comes on time.

[E3] Adjective complementation by a wh-clause.

[E3a] Adjectives with experiencer as subject.

I was unsure (of) what I should say.

[E3b] Adjectives with anticipatory it as subject.

It was unclear what they would do.

[E4] Adjective complementation by a than-clause.

She's quite a different girl than she was five years ago.

[E5] Adjective complementation by a to-infinitive clause.

[E5(i)] Bob is splendid to wait.. (Adjectives are evaluative of human conduct)

- Permits a head noun between the adjective and the infinitive. Bob must be a splendid craftsman to build this house.

- Can occur with anticipatory it and of-phrase. It was foolish of you to spend so much.

[E5(ii)] Bob is slow to react.

- The sentence corresponds to one in which the adjective becomes an adverb, while the infinitive becomes a finite verb or to one in which the adjective is followed by in and -ing..

[E5(iii)] Bob is sorry to hear it.

- Head of the phrase is an emotive adjective and the infinitive is causative.

[E5(iv)] Bob is hesitant to be with you.

- Adjective expresses volitional or modal meaning. 
[E5(v)] Bob is hard to convince.

- Subject of the sentence is identified with the unexpressed object of the infinitive clause.

[E5(vi)] The food is ready to eat.

- Infinitive can be omitted or substitute a passive infinitive clause without change of meaning.

[E5(vii)] It is important (for you) to be accurate.

- The infinitive clause may be introduced by for + subject.

[E6] Adjective complementation by an -ing participle clause.

- Without subject

Margery is busy writing letters.

- With or without subject.

It is worth (you/your) going home.

- With passive meaning.

The cartons are worth saving.

- (Optional/obligatory) insertion of preposition.

I'm busy (with) getting the house redecorated.

I'm hopeless at keeping the garden tidy.

Se trata de un sistema que pretende dar cuenta de todos los casos de complementación del adjetivo de forma similar a como se hace en el dominio verbal (cfr., Quirk et al., 1985: 1220): "Categories of complementation in adjective phrases are similar in variety to those of verb complementation". Prueba de ese afán de exhaustividad descriptiva es el hecho de incluir un modelo, la complementación mediante cláusula con than, que afecta sólo a un adjetivo, different, y, aun así, no en todos sus usos.

Estamos, por tanto, ante un modelo de referencia en la descripción completiva del adjetivo. No obstante, Hay ciertas consideraciones que conviene señalar. En principio, no todas las estructuras presentadas pueden ser consideradas modelos de complementación: ya los autores advierten en los apartados dedicados a las estructuras iniciadas con it anticipatorio ([E2b], [E3b], [E5(vii)] y [E6(ii)]) que realmente se trata de estructuras que representan al sujeto lógico de la oración extrapuesto mediante it anticipatorio. La cuestión es si la complementación del núcleo de un sintagma puede, a la vez, constituir el sujeto de la oración de la que dicho sintagma depende. Obviamente esto no es posible, por lo que hemos de considerar estos casos como secuencias sintagmáticas derivadas de una transformación temática de la oración. Evidentemente, estas distribuciones sí que responden al concepto lineal de complementación establecido en Quirk et al. (1985: 65) ("the function of a part of a phrase or clause which follows a word, and completes the specification of a meaning relationship which that word implies"), pero no se ajusta a los criterios de obligatoriedad, $o$ 
latencia, y selección por el núcleo rector de la dependencia, factores que, tal como hemos acordado, evidencian construcciones de verdadera complementación.

En relación con el llamado it anticipatorio, conviene recordar la posición expresada por Bolinger (1977: cap. 4) en el sentido de señalar que la inserción de it viene motivada por una necesaria anáfora discursiva que hace inaceptable en determinados contextos la posición de la cláusula en el lugar propio del sujeto, debiendo ésta ser extrapuesta. Sostiene este autor que en las oraciones con extraposición necesaria de la cláusula sujeto el valor referencial de it está ligado a un conocimiento referencial previo o al menor peso semántico del valor informativo del verbo. Si la extraposición de la cláusula viene obligada por la necesidad de incluir un sujeto impersonal it con referencia anafórica, tal vez habría que reflexionar sobre el verdadero valor sintáctico de la cláusula que ocupa esa posición post-adjetival. La consecuencia inmediata que podemos sacar es que, tanto la oración con sujeto clausular inicial, como la de sujeto extrapuesto, son oraciones independientes y no formas alternativas de la misma.

Sea como fuere, esta matización no cambia el criterio anteriormente expuesto según el cual la cláusula extrapuesta no tiene valor de complementación, pues no está seleccionada por el adjetivo y, por tanto no ha de ser recogida por el lexicón sintáctico; sin embargo, sí debe figurar en el corpus informativo de los DMA, cuya caracterización sintáctica de esta categoría queremos analizar, ya que se trata, en definitiva, de construcciones que el estudiante no nativo de la lengua debería conocer.

\subsection{Tipología transitiva del adjetivo}

Así pues, el sistema de estructuras definido por Quirk et al. (1985) resulta insuficiente como tipología completiva, en tanto que no cubre exclusivamente comportamientos sintácticos de unidades seleccionadas por los adjetivos. Para lograr obtener un modelo tipológico real debemos volver al principio de selección argumental de los complementos por parte de los núcleos rectores de las dependencias en los sintagmas, de forma análoga al sistema verbal. Esto nos lleva a asumir para categorías no verbales como el adjetivo una relectura del concepto de la transitividad como motor de la complementación. Es el camino elegido por muchos estudios descriptivos, que encuentran en la transitividad el marco idóneo para esa tipología completiva. En este terreno, cabe reseñar aquí la propuesta planteada por Comesaña Rincón (1998), que defiende un modelo de las posibilidades de subcategorización adjetiva basado en el reconocimiento de la capacidad transitiva para esta clase de palabra. Un importante apoyo argumental 
para su propuesta lo expresa la siguiente cita de Bolinger y Sears (1981: 85), citado en Comesaña Rincón (1998: 190):

A broader way of viewing transitivity is in terms of completeness. Does a verb in a given sense require a complement -no matter whether direct, indirect, or prepositional? From this standpoint to depend in its usual sense is transitive because we have to add on something. Similarly to be fond: Jane is fond of him, not just "Jane is fond. The incomplete *They told can be completed with either a direct object (told the story) or an indirect one (told me). To be sure is transitive just like to know: I'm sure it's ok.

La redefinición de la transitividad para dar cobijo a la complementación de los predicados no verbales no es nueva: hemos aludido a una cierta tradición de autores que la sugieren, aunque, ciertamente, no de modo explícito, tal vez por las limitaciones y condicionantes que, juiciosamente, se le reconoce. Aprestémonos a señalar que ese empeño no es gratuito, sino que obedece a una finalidad clara. Comesaña Rincón (1998: 195) observa que "las gramáticas de referencia al uso no proveen de unas etiquetas claras y distintas que reflejen el potencial completivo del adjetivo, sino que lo reducen todo al término genérico e indiferenciado de 'complementación'”. Por otra parte, señala acertadamente que los diccionarios no hacen ningún intento de "sistematización gramatical" que refleje esa capacidad completiva, probablemente motivado por la limitación, al menos, teórica, de estas unidades a ser seguidas por un grupo nominal objeto, ignorándose el hecho de que los adjetivos sí tienen esa "capacidad completiva real de Objeto, la cual viene desarrollada por grupos sintácticos paralelos a aquellos distinguidos en la esfera verbal, incluido un Grupo Nominal, aunque en una menor cuantía”. Ese reconocimiento, finalmente, abre la puerta a una plausible sistematización descriptiva lexicográfica, no sólo de los adjetivos, sino del predicado en su totalidad (verbal y no verbal).

Sin embargo, una de las mayores dificultades con que se encuentra esa propuesta de tipología 'transitiva' de los predicados no verbales viene del propio concepto de Objeto asociado con ella, Comesaña Rincón (1998: 190 y sig.) demuestra que las dificultades teóricas que enquistan este término conceptual no lo son tanto, y no impiden una cierta flexibilidad en su reconsideración. En concreto, indica este autor cuatro características de los objetos que pueden encontrar en la complementación de los adjetivos un paralelismo con la esfera verbal:

a) Conexión íntima con el elemento predicativo, conforme al carácter obligatorio de algunos de sus complementos.

b) Implicación o afectación de elementos distintos al sujeto. 
c) La no mediación de preposiciones y, por tanto, la subcategorización de grupos nominales. Se analizan los casos de like, worth y unbecoming como adjetivos que rigen grupos nominales ( $c f r$., She is like her mother; The sculpture is worth a closer study y His conduct is unbecoming an officer).

d) La realización de la función objeto por cláusulas finitas y no finitas con función nominal (cfr., Some people are glad (that) you came back so soon).

Obviamente, la elevación a sujeto por la transformación pasiva queda descartada, ya que ésa es una propiedad exclusiva del verbo y, aún así, hay que recordar que no en todos los casos funciona como test válido para identificar al objeto (cfr., These gloves fit you/"You are fitted by these gloves).

La propuesta que estamos comentando se orienta hacia la definición de una tipología completiva de la categoría adjetiva basada en una consideración de la transitividad "en términos de plenitud sintáctico-semántica" ( $c f r$., Comesaña Rincón, 1998: 190) que permita, por un lado, una clasificación de los tipos completivos más allá de la mera clasificación estructural presentada en Quirk et al. (1985), y, por otro, la búsqueda de una aplicación lexicográfica global de la complementación no verbal, ya que, como tendremos ocasión de comprobar, los diccionarios no dedican el mismo rigor que al verbo a la hora de incluir en sus páginas ese tipo de información y, cuando lo han hecho ésta ha sido poco sólida y efímera (cfr., $\left.L D O C E_{1}, 1978\right)$.

Siguiendo el exhaustivo modelo taxonómico aplicado al verbo en Garrudo Carabias (1991, 1996), Comesaña Rincón (1998: 194-5) presenta la siguiente propuesta de clasificación tipológica del adjetivo atendiendo a sus posibilidades completivas:

INTRANSITIVO. No necesitan complementación.

She was so FULL (UP) that she could not have anything else.

TRANSITIVO. Adjetivos que desarrollan una complementación mediante alguna de las siguientes categorías:

a) OBJETO (clausulares e, incluso, grupos nominales).

i) Cláusula that. Cambodians are $A W A R E$ that they are deeply insecure.

ii) Cláusula whBe CAREFUL how you bold the baby.

iii) Cláusula de infinitivo. Is be WILLING to do it?

iv) Cláusula -ing. I'm BUSY sending e-mails.

v) Grupos nominales. To be at Harvard is WORTH the effort. 


\section{b) GRUPO PREPOSICIONAL SIMPLE (adjetivos preposicionales). She's GOOD at playing chess \\ c) GRUPO PREPOSICIONAL DOBLE ("adjetivos ditransitivos preposicionales"). \\ They are THANKFUL TO me FOR everything I did. \\ ADJUNTADO. Adjetivo completado por adjunto. \\ When is your baby DUE? \\ COPULATIVO. El complemento es correferente con el sujeto de la predicación. Versace is TANTAMOUNT TO (saying) a fantasy of unrestrained opulence.}

Esta propuesta tipológica desarrolla su aplicación lexicográfica en dos estudios complementarios (Comesaña Rincón, 2001 ${ }^{\mathrm{a}}$ y 2001 ), donde se concretan las convenciones de notación pertinentes para la adscripción de los adjetivos en el lexicón, así como las correspondientes etiquetas relativas a los procesos de interacción de los modelos de comportamiento sintáctico, como la omisión, la elevación o la reversibilidad (cfr., Comesaña Rincón (2001 b 32 y sig.), y los casos identificativos de los argumentos semánticos de los actantes asociados a los adjetivos que se trata de catalogar. El resultado es sumamente interesante, pues el sistema propuesto posibilita la caracterización completiva de la categoría adjetiva en un catálogo lexicográfico articulado, principalmente, en torno al carácter transitivo de estas predicaciones y que, junto al mencionado diccionario sintáctico del verbo de Garrudo Carabias $(1991,1996)$ abre la puerta a un lexicón global de las predicaciones de la lengua inglesa. Veamos un ejemplo de estas caracterizaciones en Comesaña Rincón (2001 ${ }^{\mathrm{b}}$ : 40):

“ABUNDANT WITH 2 /PO/ ADJP (rico EN)

ABUNDANT $_{1} / \mathrm{A} /$ Reversibility

$\mathrm{S}+\mathrm{V}+\mathrm{ABUNDANT}+\mathrm{WITH}+\mathrm{PREP} \mathrm{O}+(\mathrm{A})$

$\mathrm{S}=\mathrm{NP}$, typ. LOCATIVE

The Canaries are said to be abundant with bananas"

Obviamente, esta propuesta atiende de modo plausible la necesidad de una caracterización tipológica de la complementación adjetival; sin embargo, por notables razones de espacio, no puede ser apropiada para la información gramatical de un diccionario. Su lugar corresponde al terreno del lexicón sintáctico. Un diccionario de aprendizaje tendrá que recoger lo más significativo de esa información de la forma más resumida posible; pero, a la vez, el usuario del mismo seguirá necesitando conocer también aquellas otras estructuras no argumentales de los adjetivos con valor idiosincrásico en el uso de la lengua. 


\subsection{La propuesta lexicográfica valencial: Herbst (1984)}

En el ámbito de la gramática valencial, desde la que, con mayor insistencia, se propugna la configuración de aplicaciones lexicográficas para las dependencias regidas por las unidades léxicas plenas, se pretende llegar a un criterio válido de catalogación de los rasgos completivos del adjetivo que cubra en su totalidad todos los casos que, necesariamente, han de reflejar los DMA. Herbst (1987: 42) reclama ese requisito de exhaustividad: "A valency dictionary of this kind should prove superior to general learners' dictionaries with respect to comprehensiveness of coverage: it should be one of the characteristics of a valency dictionary to include all the patterns a word permits". Reconoce, claro está, que toda esa información se encuentra con un importante problema de limitación de espacio en el cuerpo del diccionario, pero considera que el beneficio informativo sobre las palabras más frecuentes justifican suficientemente el sacrificio de otras; criterio que, por otra parte, define en gran medida la esencia de estas obras para estudiantes no nativos de la lengua.

El modelo que se desprende de la propuesta de Herbst (1984) y (1987) se basa en la clásica distinción, ya planteada en Matthews (1981), entre elementos periféricos, de libre adición, y los complementos, obligatorios y opcionales, seleccionados específicamente por la pieza léxica rectora de la dependencia, que forman la valencia de ésta, y que, por tanto, forman parte de su caracterización sintáctico-semántica.

Tal como señalábamos al referirnos a la complementación en general, la frontera entre estas tres clases de elementos no está claramente marcada y los numerosos intentos teóricos de delimitarlos no ofrecen criterios realmente concluyentes. Sin embargo, la necesidad de recoger esa distinción en los diccionarios legitima, según Herbst (1987: 33), asumir en parte ese margen de arbitrariedad:

Where the distinction between complements and peripheral elements is set up for the purposes of an EFL-dictionary it is however legitimate to consider problems of the foreign learner and lexicographical practicability as decisive factors. The function of such criteria is not to ignore the fact that there is considerable overlap between obligatory complements, optional complements and peripheral elements, but to decide borderline cases in a systematic way and thus to guarantee consistency in the elicitation of the patterns to be included in the dictionary. 
Herbst (1984: 8) considera, no obstante, un elemento fundamental la inclusión en la información valencial de los adjetivos de aquellos factores de índole semántica relevantes para el comportamiento sintáctico que se pretende reflejar (cfr., [-ANIM] useful for lexicography / [+ ANIM] useful to lexicographers), señalando la necesidad de incluir información "on the selectional restrictions of the complements in the valency description or, (...), to specify what could be called the semantic function of the complements by labels similar to those used in case grammar".

Este autor considera necesario aportar una forma de mejora de la información sintáctica de los adjetivos recogida en los diccionarios (en concreto, se refiere a $A L D$ y $L D O C E)$, donde, según señala, la extensa información sobre el verbo no se corresponde con una instrucción similar referida a la categoría adjetiva ( $c f r$., Herbst, 1984: 1): "The coverage of adjectival complementation in the $A L D$ and the $D C E$ is far less systematized than that of the verb patterns. Especially, it is not clear on what grounds the compilers have decided whether or not to specify the complementation possibilities of an adjective").

Así pues, este autor entiende probada la necesidad de marcar en los repertorios léxicos todas las posibilidades que puedan darse en la complementación obligatoria y opcional de los adjetivos. Herbst (1984: 5) aporta la evidencia de su análisis (cfr., Herbst, 1983) basado en 555 entradas de adjetivos del que se desprende que sólo se etiquetan con modelos de complementación un treinta por ciento en $L D O C E$ y, aproximadamente, un veinte por ciento en $A L D$. De ellos, los complementos preposicionales de los adjetivos señalados superan ligeramente esos mismos porcentajes ( $32 \%$ en $L D O C E$ y $21 \%$ en $A L D$ ) y, por último, se reduce a niveles mínimos en los modelos completivos con infinitivos o cláusulas ( $10 \%$ en $L D O C E$ y $4 \%$ en $A L D)$. Tampoco los ejemplos ilustran totalmente, según Herbst, las posibilidades de complementación vistas con criterios valenciales: "only 40 per cent of the prepositional complements and about 20 per cent of the infinitival and clause patterns are given in the $A L D$ and $D C E$ ". En virtud de esa crítica, la propuesta valencial de Herbst, justificada como una contribución a la mejora de las posibilidades informativas de los diccionarios en estos aspectos, se constituye como un modelo tipológico más de las posibilidades completivas del adjetivo ${ }^{2}$.

2 De reciente publicación, $A$ Valency Dictionary of English, compilado por Herbst (Herbst et al., 2004), recoge todas las aportaciones de este autor en materia de información sintáctica a la lexicografía pedagógica. 


\section{LA CODIFICACIÓN DE LA COMPLEMENTACIÓN DEL ADJETIVO EN LOS DMA}

Si se analiza el tratamiento que de la complementación verbal hacen los DMA ( $c f r$., Rizo Rodríguez, 1992), se evidencian claramente los esfuerzos que, en mayor o menor medida, los tres repertorios hacen por reflejar con detalle el comportamiento sintáctico derivado de los verbos que desarrollan estructuras oracionales distintivas. Esos comportamientos fijos se reflejan en los "patterns" codificados para su oportuna información comprimida dentro de las diferentes entradas verbales del diccionario. La evolución de esos sistemas de códigos en las sucesivas ediciones ha buscado una simplificación de los esquemas y una mejor comprensión por los usuarios, manteniendo el rigor de la idea original.

$\mathrm{Al}$ acercarnos a las características completivas de categorías no verbales comprobamos que la orientación es distinta: la información que sobre la sintaxis del adjetivo o el nombre aportan los repertorios que nos ocupan no presenta tanta variedad y rigor como los modelos verbales. Esta atención tiende a reducirse edición tras edición y, prácticamente, se limita en las últimas a la inserción ocasional de abreviaturas de proformas o referencias a la preposición usada, y no en todos los casos. En muchas ocasiones, los adjetivos no merecen para el compilador mayor atención que la que concierne a su posición, sus valores nocionales y rasgos morfológicos.

Este hecho que nos proponemos constatar, nos lleva, por el momento a la siguiente reflexión: por una parte, es sorprendente que la codificación sintáctica del adjetivo en los DMA haya ido perdiendo interés entre los editores de estas obras cuando se han ido desarrollando los estudios sobre esta categoría gramatical. Piénsese que ya el esquema oracional del adjetivo presentado por Quirk et al. (1985: 1220-31) establecía 17 categorías descriptivas (6 modelos básicos y 11 subtipos) del comportamiento completivo del adjetivo. Por otro lado, resulta sorprendente observar el rigor que, históricamente, caracteriza el tratamiento de los verbos en los DMA y el cuidado que ponen sus editores en ayudar a los usuarios no nativos a evitar en su producción los errores relacionados con esta clase de palabras (preocupación muy loable, por cierto) y, paradójicamente, abandonarlos a su suerte en muchas ocasiones con respecto al uso de otras categorías. En términos generales, entendemos que la producción lingüística puede ver amenazada su comprensibilidad tanto por factores distribucionales que afecten al verbo como al adjetivo: no está claro en virtud de qué criterio puede ser más grave un error como *They sell to people books, que *This book is easy of read. En ese sentido, la preocupación pedagógica que guía la compilación de estos diccionarios debería buscar un mayor equilibrio a favor de 
las categorías no verbales. Veamos de qué forma están tratadas esas posibilidades en los DMA que estamos describiendo.

\subsection{Los diccionarios elegidos}

Hemos considerado oportuno basar nuestro análisis en los tres DMA ingleses considerados hoy día clásicos en su género. En primer lugar, el Oxford Advanced Learner's Dictionary of Current English $(A L D)$, pionero en este tipo de obras, que a lo largo de su historia (actualmente va por su séptima edición) ha demostrado una seria preocupación por recoger de forma exhaustiva todos los modelos de complementación verbal diseñados originalmente por el fundador de la saga, el profesor A. S. Hornby, e incluidos en un interesante catálogo de "patterns" convertido en un clásico de referencia en la enseñanza del inglés (cfr., Hornby, 1954). Este lexicógrafo promovió, en colaboración con otros autores las sucesivas ediciones del Advanced Learner's Dictionary, hasta la edición definitiva por parte de la editorial Oxford University Press ${ }^{3}$. En segundo lugar, el que se considera el gran competidor por muchos años de la obra iniciada por Hornby, el proyecto de la casa Longman que en 1978 editó el profesor P. Procter, el Longman Dictionary of Contemporary English. Hemos tomado como referencia las tres primeras ediciones ( $L D O C E_{1}, 1978 ; L D O C E_{2}, 1987$ y $\left.L D O C E_{3}, 1995\right)$, ya que en todas ellas se reflejan cambios cualitativos y cuantitativos en su tratamiento gramatical que deben ser tenidos en cuenta. Por desgracia, en las ediciones más recientes del diccionario los códigos gramaticales se han ido reduciendo, cuando no omitiendo, dejándose en muy segundo plano en la información aportada.

Por último, es imprescindible tener en cuenta la notable aportación de un tercer proyecto editorial de características muy novedosas, el diccionario editado en 1987 por J. M. Sinclair, el COBUILD English Language Dictionary (COBUILD, 1987). Aunque del ambicioso proyecto "COBUILD" han ido apareciendo en los últimos años numerosas publicaciones, no sólo lexicográficas,

3 La saga comienza con la publicación en 1942 de Idiomatic and Syntactic English Dictionary, editado por A. S. Hornby, E. V. Gatenby, y H. Wakefield, reimpreso, a su vez, en 1948 en Oxford University Press como A Learner's Dictionary of Current English, y en 1952 con el título The Advanced Learner's Dictionary of Current English), Tokyo, Kaitakusha; reeditado en 1957 como The Oxford Advanced Learner's Dictionary of Current English, London, Oxford University Press. Ésta es la considerada primera edición a la que siguieron dos más, en 1963 y 1974, dirigidas por Hornby. En nuestro análisis nos centraremos en la cuarta edición del diccionario, dirigida por A. P. Cowie (ALD, 1989). 
sino varias gramáticas y conocidos métodos de inglés, se mantiene esta edición como la obra de referencia y será la que abordaremos en nuestro análisis.

\subsubsection{El tratamiento del Oxford Advanced Learner's Dictionary}

Este diccionario, en su cuarta edición (ALD, 1989), recoge muy brevemente en su guía una referencia a la complementación de nombres, adjetivos y verbos que resultan "incomplete and ungrammatical without a following prepositional phrase or non-finite construction"; se refiere, además, a los dos tipos, obligatorio y opcional ("but highly predictable") y, tras recordar que estos comportamientos, en lo que se refiere al verbo, quedan recogidos en las 17 páginas explicativas de los códigos (págs. 1554-70), indica que en el diccionario "complementation is shown by means of a pattern in bold print after the grammatical code(s)" (pág. 1572). Comprobamos por los ejemplos que aporta que esos "grammatical codes" a los que alude se refieren a los datos morfológicos de las categorías no verbales (sólo la complementación verbal se encuentra codificada): "tanta·mount (...) adj [pred] to sth...” (pág. 1572). Es decir, la complementación no verbal es presentada, de modo secundario, mediante abreviaturas que acompañan a los códigos morfológicos. En concreto, se aportan las cuatro etiquetas siguientes:

$\sim$ adjetivo o nombre seguido de (el uso de paréntesis indica opcionalidad):

$\sim$ (that): cláusula that.

$\sim$ (of/about/from/etc.): la preposición que se indica.

$\sim$ (to do sth.): un infinitivo con to.

$\sim$ (doing sth.): un verbo en la forma -ing.

En muchas ocasiones, no se ofrece la información sobre el tipo completivo, éste es sólo sugerido por los ejemplos ilustrativos.

\subsubsection{La complementación del adjetivo en los diccionarios "Longman"}

El caso más evidente de esta falta de interés por reflejar la sintaxis no verbal a la que nos estamos refiriendo lo encontramos en la trayectoria de LDOCE. Efectivamente, resulta significativo rastrear las primeras tres ediciones de este diccionario: En su primera edición (LDOCE1, 1978) se recogían, entre otros rasgos gramaticales, cuatro códigos de complementación adjetiva:

[F] Adjetivo en posición predicativa seguido de:

[F3] Infinitivo.

[F5] Cláusula that.

[F6] Cláusula wh-seguida de un infinitivo con to.

$[\mathrm{F}(\mathrm{for})] \quad$ La preposición que se incluye (el paréntesis indica opcionalidad). 
En la segunda edición $\left(L D O C E_{2}, 1987\right)$ los códigos alfanuméricos desaparecen y sólo se ofrecen tres modelos de estructuras que pueden seguir al adjetivo o al nombre:

$$
\begin{array}{ll}
{[+ \text { with } /(\text { about })]} & \begin{array}{l}
\text { Debe o puede (paréntesis) ir seguido de la preposición } \\
\text { incluida. }
\end{array} \\
{[+ \text { to-v }]} & \text { Seguido por un infinitivo. } \\
{[+ \text { that }]} & \text { Seguido por una cláusula that. }
\end{array}
$$

Por fin, la tercera edición $\left(L D O C E_{3}, 1995\right)$, que aporta etiquetas sintácticas sobre la posición de los adjetivos y adverbios, "[only before noun], [only after noun], etc.” (xix), no ofrece ninguna referencia específica sobre el régimen clausular o preposicional del nombre o el adjetivo. Sólo en su página resumen de los códigos usados en el diccionario se da a entender que en las entradas de algunas palabras (sin referencia específica a la categoría de éstas) podemos encontrar la etiqueta referente a la preposición ("[+ between], $[+$ about]"), cláusula that ("sure (that)"), o cláusula de infinitivo ("resolve to do sth.") que las completan.

\subsubsection{El diccionario COBUILD}

COBUILD, por un lado, fiel a su idea editorial de no conceder utilidad lexicográfica a rasgos del adjetivo que vayan más allá de los nocionales y posicionales ( $c f r$., Krishnamurthy, 1987: 67) y, en general, por su línea gramatical (cfr., Sinclair, 1987: 107: "a means of recording structure must be devised which depends as little as posible on a theory. The more superficial, the better.") centrada ésta, además, en el uso y la gramática del texto, no recoge ningún modelo específico de complementación para el adjetivo o el nombre. A las abreviaturas usadas con estas clases de palabra referentes a los aspectos morfológicos, COBUILD aporta sólo unas etiquetas indicativas de la estructura que puede o debe seguir a la palabra descrita. Esas abreviaturas son definidas en sus entradas correspondientes (special entries) como elementos completivos del verbo, sólo de modo secundario se aplican a otras palabras. No hay en COBUILD, podemos concluir, marcadores de modelos específicos para las categorías no verbales. Los indicadores usados son los siguientes:

: Palabra seguida de:

-ING

TO -INF

Verbo en la forma -ing.

(PREP...) Preposición obligatoria en ese contexto. Se indica la preposición.

REPORT CL Cláusula wh-o that. 
Teniendo en cuenta las aportaciones realmente novedosas que este proyecto editorial incluye en su diccionario, Comesaña Rincón (1989) lamenta que no se haya aprovechado la ocasión que su formato y nuevos enfoques lexicográficos brindaban para una mejor atención a la sintaxis del adjetivo. Fiel a su propuesta de un estudio taxonómico de esta clase de palabra paralelo al del verbo, la crítica de este autor se centra en los siguientes cuatro aspectos:

a) La no consideración de características completivas aplicadas al verbo, como la (in)transitividad, o de otros aspectos sintáctico-semánticos, como la reciprocidad, de los que sí son objeto de atención los verbos.

b) Confusión, sin más, de los participios de pasado de algunos verbos con los adjetivos en las explicaciones de las special entries y, a la vez, la no distinción entre adjetivos en -ed y verbos reales.

c) No diferencia entre elementos completivos obligatorios y opcionales.

d) No distinción de casos de homonimia léxica (like preposición / like adjetivo) con entradas diferentes.

\section{LA COMPLEMENTACIÓN DEL ADJETIVO EN LOS DICCIONARIOS: VALORACIONES Y PROPUESTAS DE MEJORAS}

Una de las diferencias esenciales entre el tratamiento de la gramática en un lexicón sintáctico y el de un diccionario radica en el carácter canónico de las estructuras recogidas en el primero, frente a la vocación práctica de éste. En efecto, mientras el lexicón trata de reflejar los modelos sintácticos de las oraciones no marcadas, en su estadio pre-transformacional, un diccionario dirigido a compendiar los usos gramaticales de los lexemas que define no puede conformarse con codificar las formas prototípicas: su tipología debe dar cuenta también de las formas marcadas temáticamente o derivadas por motivos de naturaleza pragmática, que tengan valor como fórmulas sintagmáticas idiosincrásicas, no como meras estructuras alternativas, que el aprendiz del idioma debe saber usar. Así, por ejemplo, en el caso de adjetivo seguido de infinitivo en estructura oracional iniciada por it, como en it would be fatal to do that, la cláusula de infinitivo no tiene el valor de complemento que encontramos en this book is useful to register the customers; no hay por qué recogerlo, pues, en el lexicón sintáctico, pero el diccionario de aprendizaje sí está obligado a testimoniar su uso, toda vez que su naturaleza de sujeto extrapuesto se encuentra condicionada por factores discursivos (como la referencia previa) que, según sea el caso, pueden hacer inaceptable la alternativa to do that would be fatal y hacer necesaria la extraposición (cfr., Bolinger, 1977: cap. 4). A pesar de ello, el usuario ha de ser informado de la distinción entre formas canónicas de complementación y fórmulas eventuales condicionadas por el uso o, como hemos señalado, por 
factores discursivos. La codificación que proponemos, pues, debe incluir no sólo los complementos reales seleccionados por el adjetivo, sino aquellas otras construcciones que conlleven un uso específico asociado a éste. En todo caso, será necesario distinguir su naturaleza completiva en el código empleado. A este fin, bastará con marcar el código con una $\mathrm{C}$ mayúscula previa en los casos de complementación.

Por otro lado, cabe señalar la importancia que tendría, como primera medida en nuestro planteamiento descriptivo, el poder hacer uso de un formato de presentación cercano al empleado por el diccionario COBUILD, mediante el cual quedara desligada la información principal semántica de cada lema de la codificación gramatical y los oportunos ejemplos ilustrativos, que podrían tener su sitio en una columna paralela, gráficamente aislados del resto, aunque convenientemente relacionados con las definiciones asociadas.

El tratamiento que proponemos para el adjetivo en los diccionarios learner's no pretende tanto crear elementos descriptivos nuevos, como la aplicación de forma exhaustiva de todos los criterios tipológicos afines a esa categoría en todas las entradas de la misma. Cada lema adjetivo debe ser etiquetado para el reconocimiento, no sólo de sus rasgos morfológicos y posicionales (aspecto que las obras que nos ocupan cuidan sin excepción), sino, cuando sea el caso, también de los referidos a sus posibilidades completivas a través del correspondiente sistema de códigos, tal como se describen las entradas verbales. En ese sentido, los códigos que proponemos no se alejan de los empleados, por ejemplo, en $A L D$, que nos parecen bastante cómodos para el usuario, pero estimamos oportuno añadir algunos más que cubran modelos que los diccionarios consultados no aportan+.

Por otro lado, consideramos necesario marcar el modelo sintáctico de cada código con una indicación sobre su valor como complementación; es decir, como argumento seleccionado por el propio adjetivo. Esa naturaleza, codificada ,como se ha señalado antes, con una $\mathbf{C}$ colocada delante del código correspondiente, se distingue de otras secuencias sintagmáticas no argumentales, cuyo valor idiosincrásico requiere su inclusión codificada en la información de los adjetivos. Los complementos opcionales, entendiendo por tales aquellos que, por predecibles, pueden no estar contextualmente presentes, se indicarán, como suele ser habitual, mediante el uso de paréntesis; cualquier otro elemento completivo necesario para la pieza léxica sólo será considerado elidible en virtud del

4 Véanse también, a este respecto, Alexander \& Matthews (1964) y Bridgeman et al., (1965). 
principio de latencia. Asimismo, todos los usos distintivos descritos deben llevar unido su ejemplo ilustrativo, que nunca debe sustituir al código, sino completar su información. La codificación propuesta podría tener la siguiente forma:

$\mathrm{C}[\mathrm{at}$; for; etc.] Complementación mediante un grupo preposicional encabezado por la preposición que se indica.

She was mad at her sister.

C[that] Complementación mediante una cláusula that.

He was confident that she would come.

C[that+subj/should $]$ Atribuye a la cláusula completiva con that la necesidad de llevar un verbo en subjuntivo o con el auxiliar should. En su defecto, cualquier modo verbal es correcto.

She is anxious that the telephone ring/should ring

[it...that] Secuencia sintagmática con cláusula that sujeto pospuesta al adjetivo e inicio de oración con it anticipatorio.

It was obvious that they had gone.

[it... (to sth/sb) that] Variante de la anterior, con inserción opcional de un grupo nominal iniciado por la preposición to antes de la cláusula that.

It was crucial to my future that they accepted my offer.

C[wh-] El adjetivo selecciona una complementación mediante una cláusula iniciada por un elemento wh.

She was unsure why they needed that.

C[of, about, etc. wh-] La cláusula wh va precedida de la preposición que se indica.

He was pleased about what she had said.

$\mathrm{C}[$ wh $+t o-\mathrm{v}]$ La cláusula completiva wh va seguida de un infinitivo.

She wasn't sure whom to trust.

$\mathrm{C}[t o-\mathrm{v}]$ Complementación del adjetivo mediante una cláusula de infinitivo.

They were ready to leave.

[it... to-v] Secuencia sintagmática iniciada por it anticipatorio.

It isn't hard to do.

[it... (for sth/sb) to-v] Igual que la anterior, con inserción opcional del sujeto del infinitivo mediante la preposición for.

It was easy for me to do. 
C[it...(of) to-v] Alternativa a la construcción anterior que permite la inserción de una 'of-phrase', siendo ésta complemento del adjetivo usado.

It was kind of your friends to come.

Attrib $+\mathrm{C}[t o-\mathrm{v}]$ Complementación por cláusula de infinitivo de un adjetivo en posición atributiva.

It is an easy job to do.

C[V-ing] Muestra la posibilidad de complementación del adjetivo mediante un verbo en la forma de -ing.

It was worth trying.

C[at, about, etc.V-ing] Muestra la necesidad de usar la preposición que se indica con la forma verbal en -ing. He's good at playing football.

$\mathrm{C}$ [at, etc. (poss) V-ing] Inserción de un posesivo antes del verbo en -ing. They were glad at my coming home.

C[than] Complementación del adjetivo mediante cláusula than.

She is quite different than she was years ago.

\section{CONCLUSIONES}

Las ediciones más recientes de los DMA han simplificado notablemente los códigos de información gramatical en virtud de una mayor economía de espacio, suprimiendo elementos considerados de menor interés para el usuario y manifestando una línea editorial común encaminada a minimizar todos esos datos; se salvan, casi exclusivamente, los relativos a la morfología flexiva y léxica y los rasgos completivos reciben una atención menor, excepción hecha del caso del verbo que sigue obteniendo la máxima atención, incluso en tratamientos reducidos de la gramática. El resto de las clases de palabra no parece merecer en estos repertorios un tratamiento similar, a pesar de que su comportamiento sintáctico, como hemos visto en nuestro resumen teórico, no se aleja demasiado del de esa categoría, salvo, claro está, en su número y frecuencia de aparición, mucho menor en el caso de los adjetivos predicativos complementados. No obstante, el tratamiento dado resulta insuficiente al no cubrir todos los casos de complementación y no aportar la información de forma exhaustiva en todas las entradas de adjetivos con esas posibilidades. Se aprecia, asimismo, un gran contraste entre la extensa gama de modelos de codificación ideados para los verbos y la escasez de los destinados para los adjetivos.

En el caso de COBUILD, resulta doblemente decepcionante observar que, pese a su innovador y cómodo formato gráfico, no se aprovecha éste para una más amplia y mejor presentación de los aspectos gramaticales en su 'extra 
column'; recuérdese a este respecto la intención del equipo editorial de mantener su gramática en términos superficiales (Sinclair, 1987: 107). Son escasas, pues, las posibilidades de ver aumentar esa carga de información tan importante, no sólo para la producción lingüística de los estudiantes, también de enorme utilidad para los profesores no nativos, como muy acertadamente recuerda Herbst (1984: 6):

One should not forget that the ALD and the DCE [LDOCE] are not only useful tools for the foreign learner in his or her own language production, but that they are also of great importance to the foreign teacher of English, who will consult the dictionary in order to judge the acceptability of a construction used by a student.

Hoy día, la catalogación lexico-sintáctica de la lengua inglesa parece estar orientándose hacia obras complementarias de carácter marcadamente gramatical como son los diccionarios de valencias y los diccionarios sintácticos. Hemos aludido en varias ocasiones al Diccionario sintáctico... de Garrudo Carabias (1991, 1996), hasta el momento, único repertorio específico sobre el verbo inglés y las estructuras que de él dependen. Actualmente se están llevando a cabo estudios para aplicar el mismo análisis descriptivo a otras categorías no verbales que, eventualmente, han de dar lugar a un gran "lexicon" del predicado inglés, catálogo gramatical de gran envergadura e instrumento de consulta y trabajo de indudable interés para estudiantes y profesores.

Sin embargo, entendemos que, además de estas notables alternativas, la obra básica de consulta debe seguir siendo el diccionario manual de aprendizaje, para el que nos empeñamos en reclamar un tratamiento, sin excepciones, de las posibilidades gramaticales prácticas que los usuarios tienen derecho a recibir. Un adecuado adiestramiento por parte de los profesores en el aprovechamiento de esa información, unido a un esfuerzo de imaginación y buena voluntad por parte de los editores, redundaría en la devolución al aparato gramatical de los repertorios léxicos de la dignidad que en otros aspectos les caracteriza y, sobre todo, la utilidad que como instrumentos pedagógicos éstos merecen.

Del mismo modo que los patrones verbales de los diccionarios de la serie LDOCE están adecuadamente basados en un esquema tipológico (Quirk et al., 1972), conviene asumir con carácter general un marco descriptivo que propicie modelos claros de tipificación codificada de los comportamientos sintácticos esperables de la categoría adjetiva. Hemos revisado tres modelos distintos que nos pueden aportar puntos de encuentro para ese objetivo. Por una parte, el modelo del grupo de Quirk (Quirk et al., 1985) ofrece una descripción exhaustiva de las estructuras completivas de los sintagmas adjetivales, tanto de los casos de complementación propiamente dicha, como de aquellas otras secuencias 
sintagmáticas completivas que un estudiante no nativo debe conocer. La tipología transitiva (Comesaña Rincón, 1998, 2001 a y 2001 ) aporta criterios muy útiles para la clasificación cuantitativa y cualitativa de la capacidad completiva del adjetivo; la inclusión en la misma de factores de índole semántica que inciden en la configuración sintáctica la acercan a la tercera de las tres fórmulas a que nos estamos refiriendo, la de Herbst (1984), cuya tipificación de las valencias adjetivales completa el ámbito de búsqueda de la forma idónea de aplicación lexicográfica distintiva y necesaria para la información de los usuarios de DMA y su adecuación a sus limitadas condiciones de espacio.

Los equipos editoriales que dirigieron los primeros proyectos de DMA perseguían un claro fin didáctico al querer poner en manos de los estudiantes del idioma un instrumento capaz de cubrir sus necesidades en cuanto a los aspectos léxico-semánticos, pero teniendo en cuenta, a la vez, aquellos otros de naturaleza morfosintáctica que ineludiblemente contribuyen a la configuración léxica de las piezas registradas, toda vez que su correcto uso y selección gramatical participa de modo relevante en la codificación y descodificación de los textos. $\mathrm{La}$ argumentación teórica en torno al tratamiento que esos factores gramaticales debían tener en el cuerpo del diccionario transcurrió por senderos presididos por criterios de gran rigor científico. Sin embargo, toda la preocupación teórica giraba alrededor de la complementación verbal, dedicándosele mucha menos atención a los aspectos completivos de los adjetivos y los nombres. Desde una perspectiva tradicional, resulta comprensible esa disparidad de tratamiento; no olvidemos que en los momentos en que aparecen $A L D$ y $L D O C E$ el término "complementación" se asocia, casi exclusivamente, con la de la categoría verbal, siendo ésta responsable de la tipología oracional derivada de su sintaxis.

No obstante, según hemos pretendido reflejar en los apartados dedicados a analizar el estado actual de las cuestiones relativas a la complementación, el marco teórico sobre las capacidades completivas del adjetivo y el nombre goza actualmente de un nivel de madurez suficiente como para desear que tengan un sitio en el aparato informativo de los diccionarios pedagógicos. Indudablemente, la orientación hacia la producción de catálogos sintácticos de tipo "lexicon" es un reto importante, a la luz de esos mismos criterios teóricos que hemos revisado, pero su aplicación a pequeña escala en la herramienta básica del diccionario de aprendizaje nos parece un desafío que merecería la pena aceptar. 


\section{REFERENCIAS BIBLIOGRÁFICAS}

ALEXANDER, A. y MATTHEWS, P.H., "Adjectives before that-clauses in English", Linguistic Research Project, Bloomington, Indiana University Linguistic Club, (1964), pp. 1-28.

BOLINGER, D., Meaning and Form, London, Longman, 1977.

BOLINGER, D. y SEARS, D. A., Aspects of Language, New York, Harcourt Brace Jovanovich, Inc., 1981.

BRIDGEMAN, L.I., DILLINGER, D., HIGGINS, C., SEANAB, P.D. y SHANK, F.A. "Further classes of adjectives", Linguistic Research Project, Bloomington, Indiana University Linguistic Club (1965), 1-23.

COMESAÑA RINCÓN, J., "El tratamiento del adjetivo inglés en el proyecto COBUILD", Actas del VI Congreso Nacional de Lingüistica Aplicada (AESLA), Santander: Universidad de Cantabria, (1989), pp.183-92.

COMESAÑA RINCÓN, J., "La transitividad adjetiva: hacia una tipología completiva del adjetivo en inglés" en Transitivity Revisited, ed. M. R. Martínez Vázquez, Huelva, Servicio de publicaciones de la Universidad de Huelva, 1998, pp.187-199.

COMESAÑA RINCÓN, J., Introduction to the Morphosyntax of the English Simple Sentence, Granada, Comares, 2000.

COMESAÑA RINCÓN, J., "Decoding and encoding grammatical information in adjectival entries: the basics", Atlantis, 23, $1\left(2001^{2}\right)$, pp. 27-40.

COMESAÑA RINCÓN, J., "Decoding and encoding grammatical information in adjectival entries: processes and cases" en Atlantis, 23, 2 (2001 $)$, pp. 31-48.

COWIE, A. P., English Dictionaries for Foreign Learners: A History, Oxford, Oxford University Press, 1999.

DODD, W. S., "Tendencias en la lexicografía anglosajona: los diccionarios monolingües para usuarios extranjeros" en Aspectos de lexicografía contemporánea, ed. H. Hernández Hernández, Barcelona, Biblograf, 1994, pp. 39-59.

ESCANDELL VIDAL, M. V., Los complementos del nombre, Madrid, Arco/Libros S.L., 1995.

FERNÁNDEZ LEBORANS, M. J., Los sintagmas del español. I. El sintagma nominal, Madrid, Arco/Libros, S.L., 2003.

GARRUDO CARABIAS, F., Diccionario sintáctico del verbo inglés, 2 vols., Barcelona, Ariel, 1991, 1996. 
HERBST, T., Untersuchungen zur Valenz englisher Adjektive und ibrer Nominalisierungen. Tübingen, Gunter Narr, 1983.

HERBST, T., "Adjective complementation. A valency approach to making EFL dictionaries”, Applied Linguistics, vol. 5, 1 (1984), pp. 1-11.

HERBST, T., "A proposal for a valency dictionary in English" en A Spectrum of Lexicography, ed. R. F. Ilson, Amsterdam, John Benjamins Publishing Company, 1987, pp. 29-47.

HERBST, T., HEATH, D., ROE, I. F. y GOTZ, D., A Valency Dictionary of English: A Corpus-Based Analysis of the Complementation Patterns of English Verbs, Nouns and Adjectives. Berlin, de Gruyter, 2004.

HORNBY, A. S., A Guide to Patterns and Usage in English, London, Oxford University Press, 1954.

HUDDLESTON, R., An introduction to the Grammar of English, Cambridge, Cambridge University Press, 1984.

KRISHNAMURTHY, R., "The process of compilation" en Looking up. An Account of the COBUILD Project in Lexical Computing, ed. J. Sinclair, London and Glasgow, Collins, 1987, pp. 62-86.

LEECH, G. y SVARTVIK, J., A Communicative Grammar of English, London, Longman, 1975.

MATTHEWS, P. H., Syntax, Cambridge, Cambridge University Press, 1981.

QUIRK, R. y GREENBAUM, S., A University Grammar of English, London, Longman, 1973.

QUIRK, R., GREENBAUM, S., LEECH G. y SVARTVIK, J., A Grammar of Contemporary English, London, Longman, 1972.

QUIRK, R., GREENBAUM, S., LEECH G. y SVARTVIK, J., A Comprehensive Grammar of the English Language, London, Longman, 1985.

RIZO RODRÍGUEZ, A. J., "Los códigos gramaticales en los diccionarios monolingües ingleses de aprendizaje", Revista de la Facultad de Humanidades de Jaén Vol. 1, Tomo 1, Granada, Universidad (Servicio de publicaciones), (1992), pp. 99-130.

SINCLAIR, J., "Grammar in the dictionary" en Looking up. An Account of the COBUILD Project in Lexical Computing, ed. J. Sinclair, London and Glasgow, Collins, 1987, pp. 104-115. 


\section{Diccionarios citados}

Collins COBUILD English Language Dictionary, (First Edition), eds., J. Sinclair, G. F. Hanks, R. Moon y P. Stock, London and Glasgow, Collins, 1987. Sigla: COBUILD

Longman Dictionary of Contemporary English, (First Edition), ed. P. Procter, London, Longman, 1978. Sigla: LDOCE 1

Longman Dictionary of Contemporary English, (Second Edition), eds. D. Summers, y M. Rundell, London, Longman, 1987. Sigla: LDOCE2

Longman Dictionary of Contemporary English, (Third Edition), ed. D. Summers, London, Longman, 1995. Sigla: $L_{D O C E_{3}}$

Oxford Advanced Learner's Dictionary of Current English (Fourth edition), ed. A. P. Cowie, London: Oxford University Press, 1989. Sigla: ALD

Manuel Ramos Cortés

mramosc@supercable.es

IES Joaquín Turina (Sevilla)

Avda. de Kansas city, s/n.

41007 Sevilla (España).
Fecha de recepción: 4/10/2006

Fecha de aceptación: 24/11/2006 